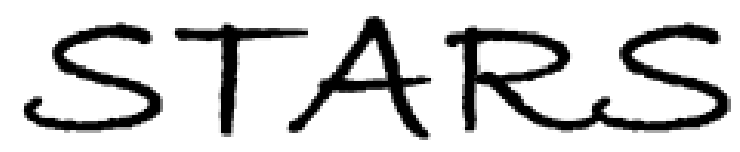

University of Central Florida

STARS

$1-1-2007$

\title{
Doping level dependence of electron irradiation-induced minority carrier diffusion length increase in Mg-doped GaN
}

O. Lopatiuk-Tirpak

University of Central Florida

L. Chernyak

University of Central Florida

Y. L. Wang

F. Ren

S. J. Pearton

See next page for additional authors

Find similar works at: https://stars.library.ucf.edu/facultybib2000

University of Central Florida Libraries http://library.ucf.edu

This Article is brought to you for free and open access by the Faculty Bibliography at STARS. It has been accepted for inclusion in Faculty Bibliography 2000s by an authorized administrator of STARS. For more information, please contactSTARS@ucf.edu.

\section{Recommended Citation}

Lopatiuk-Tirpak, O.; Chernyak, L.; Wang, Y. L.; Ren, F.; Pearton, S. J.; and Gartsman, K., "Doping level dependence of electron irradiation-induced minority carrier diffusion length increase in Mg-doped GaN" (2007). Faculty Bibliography 2000s. 7373.

https://stars.library.ucf.edu/facultybib2000/7373

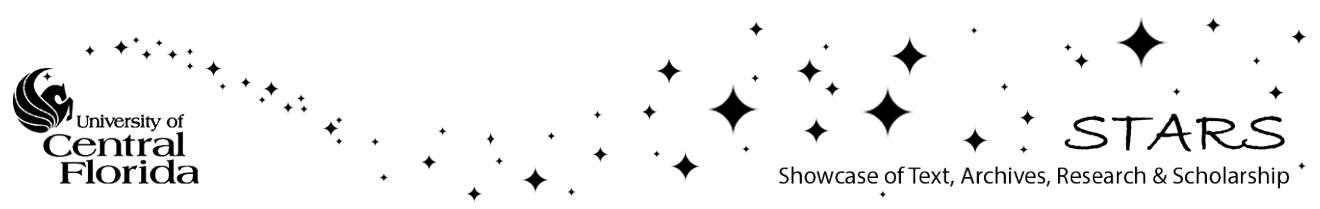


Authors

O. Lopatiuk-Tirpak, L. Chernyak, Y. L. Wang, F. Ren, S. J. Pearton, and K. Gartsman 


\section{Doping level dependence of electron irradiation-induced minority carrier diffusion length increase in Mg-doped GaN}

Cite as: Appl. Phys. Lett. 91, 092107 (2007); https://doi.org/10.1063/1.2776866

Submitted: 20 July 2007 . Accepted: 07 August 2007 . Published Online: 27 August 2007

O. Lopatiuk-Tirpak, L. Chernyak, Y. L. Wang, F. Ren, S. J. Pearton, and K. Gartsman

ARTICLES YOU MAY BE INTERESTED IN

Minority carrier diffusion length in GaN: Dislocation density and doping concentration dependence

Applied Physics Letters 86, 052105 (2005); https://doi.org/10.1063/1.1861116

Electron beam-induced increase of electron diffusion length in $p$-type GaN and AIGaN/GaN superlattices

Applied Physics Letters 77, 875 (2000); https://doi.org/10.1063/1.1306910

Minority carrier diffusion length and lifetime in GaN

Applied Physics Letters 72, 3166 (1998); https://doi.org/10.1063/1.121581
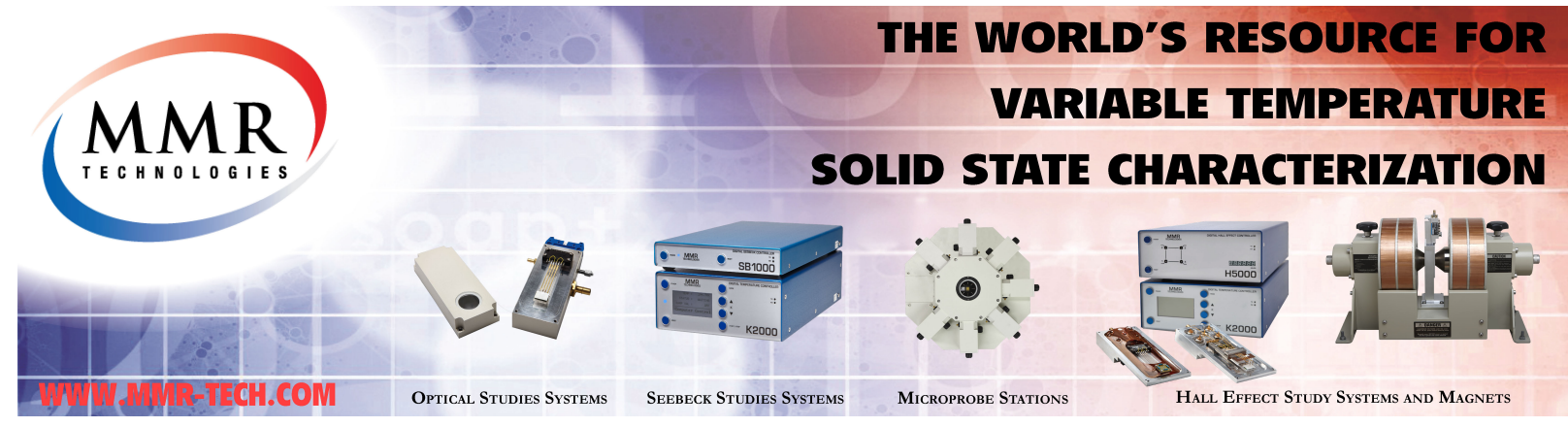


\title{
Doping level dependence of electron irradiation-induced minority carrier diffusion length increase in Mg-doped GaN
}

\author{
O. Lopatiuk-Tirpak and L. Chernyak ${ }^{\text {a) }}$ \\ University of Central Florida, Orlando, Florida 32816-2385 \\ Y. L. Wang, F. Ren, and S. J. Pearton \\ University of Florida, Gainesville, Florida 32611 \\ K. Gartsman \\ Weizmann Institute of Science, Rehovot 76100, Israel
}

(Received 20 July 2007; accepted 7 August 2007; published online 27 August 2007)

\begin{abstract}
The electron irradiation-induced increase of minority carrier diffusion length was studied as a function of hole concentration in $\mathrm{Mg}$-doped $\mathrm{GaN}$. Variable-temperature electron beam induced current measurements yielded activation energies of 264, 254, 171, and $144 \mathrm{meV}$ for samples with hole concentrations of $2 \times 10^{16}, 9 \times 10^{16}, 3 \times 10^{18}$, and $7 \times 10^{18} \mathrm{~cm}^{-3}$, respectively. This carrier concentration dependence of the activation energy for the effects of electron irradiation was found to be consistent with $\mathrm{Mg}$ acceptors, indicating the involvement of the latter levels in the irradiation-induced diffusion length increase. (c) 2007 American Institute of Physics.
\end{abstract}

[DOI: $10.1063 / 1.2776866]$

It has been demonstrated earlier that irradiation of wide band gap semiconductors ( $p-\mathrm{GaN}$ and $p-\mathrm{ZnO}$, in particular) containing deep electron traps with low energy electron beam can be used to significantly enhance minority carrier diffusion length $(L)$ and lifetime $(\tau) .{ }^{1-4}$ Furthermore, evidence suggests that this enhancement can be translated to the improved performance of GaN- and $\mathrm{ZnO}$-based UV photodetector diodes, which rely on minority carrier transport for their functionality. ${ }^{5,6}$

The mechanism responsible for the irradiation-induced enhancement of minority carrier lifetime and diffusion length has been suggested to involve the nonionized acceptor levels, located several hundred $\mathrm{meV}$ from the top of the valence band. ${ }^{7}$ These levels play an integral role in the recombination of nonequilibrium electron-hole pairs and may act as metastable electron traps. Since the filled traps are no longer available for recombination, the increase in their concentration due to continuous excitation by the electron beam leads to a reduction in recombination rate, implying greater lifetime of nonequilibrium electrons in the conduction band. Because carrier lifetime is directly related to the diffusion length $\left[L=(D \tau)^{1 / 2}\right.$, where $D$ is carrier diffusivity] and given the fact that the activation energy of the irradiation effects has been found to correlate to that of the $\mathrm{Mg}$ acceptor, ${ }^{2}$ such a mechanism provides a feasible explanation for the previously observed increase of the diffusion length under electron irradiation.

Further evidence of the involvement of $\mathrm{Mg}$ levels has been recently demonstrated by a series of cathodoluminescence measurements aimed at investigating the role of the doping level on the increase of lifetime of nonequilibrium carriers. ${ }^{8}$ It was shown that the activation energy of this phenomenon is strongly dependent on the concentration of majority carriers. In this letter, we present a complementary study of minority carrier diffusion length increase as a function of acceptor concentration. Unlike in the previous work,

\footnotetext{
${ }^{a)}$ Electronic mail: chernyak@physics.ucf.edu
}

where the increase in lifetime was deduced from the decay of cathodoluminescence intensity, here, the increase of $L$ was measured directly using the electron beam induced current (EBIC) method.

Experiments were conducted on four commercially available 3-4 $\mu \mathrm{m}$ thick GaN epitaxial films (TDI, Inc.) doped with different concentrations of magnesium. The net hole concentrations $p$ were determined by Hall effect measurements and are shown in Table I. EBIC measurements were conducted in situ in the Philips XL30 scanning electron microscope (SEM) under the accelerating voltage of $20 \mathrm{kV}$.

The rectifying contacts were prepared by electron beam evaporation of $\mathrm{Pt} / \mathrm{Au}$, following formation of Ohmic contacts using alloyed $\mathrm{Ni} / \mathrm{Au}$. This process produced excellent diodes, with on/off ratios of $>10^{8}$ at $+10 \mathrm{~V} /-5 \mathrm{~V}$. EBIC measurements were performed in a line-scan mode, where the induced current is measured as a function of distance of the SEM beam from the Schottky barrier (see Ref. 9 for a detailed description of EBIC method). After initial acquisition, the beam was allowed to scan over the same line on the sample surface, while additional measurements were taken intermittently to monitor the increase of $L$.

The SEM is also equipped with a hot stage and an external temperature controller (Gatan) allowing for temperature-dependent experiments. The rates of irradiationinduced diffusion length increase were measured at tempera-

TABLE I. Room-temperature hole concentrations and activation energies $\left(\Delta E_{A, I}\right)$ for the electron irradiation-induced diffusion length increase in GaN:Mg.

\begin{tabular}{ccc}
\hline \hline Sample & $\begin{array}{c}\text { Hole } \\
\text { Concentration } \\
\left(\mathrm{cm}^{-3}\right)\end{array}$ & $\Delta E_{A, I}(\mathrm{meV})$ \\
\hline $\mathrm{A}$ & $2 \times 10^{16}$ & $264 \pm 43$ \\
$\mathrm{~B}$ & $9 \times 10^{16}$ & $254 \pm 24$ \\
$\mathrm{C}$ & $3 \times 10^{18}$ & $171 \pm 11$ \\
$\mathrm{D}$ & $7 \times 10^{18}$ & $144 \pm 11$ \\
\hline \hline
\end{tabular}




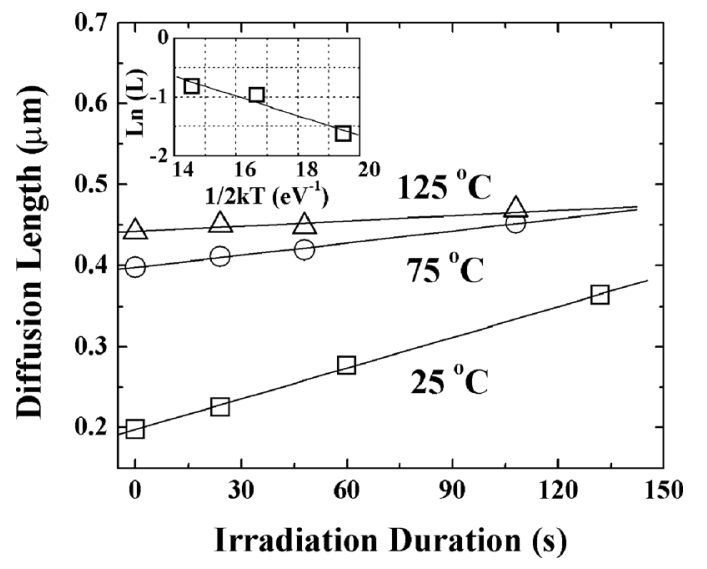

FIG. 1. Increase of minority carrier diffusion length in sample A under continuous irradiation by the electron beam at various temperatures. Inset: temperature dependence of the preirradiation values of diffusion length and the fit with Eq. (1).

tures ranging from 25 to $125^{\circ} \mathrm{C}$. At each temperature, the measurements were performed on a previously unexposed location.

Figure 1 shows the linear increase of the minority electron diffusion length in sample $\mathrm{A}$ as a function of irradiation duration, observed at different temperatures. The temperature dependence of $\mathrm{L}$ prior to electron injection was found to be similar to that observed earlier for GaN (Ref. 9) and can be described using the following relationship:

$$
L=L_{0} \exp \left(-\frac{\Delta E_{A, T}}{2 k T}\right),
$$

where $L_{0}$ is a scaling constant, $\Delta E_{A, T}$ is the thermal activation energy, $k$ is the Boltzmann constant, and $T$ is the sample temperature. This dependence allows us to estimate the activation energy for thermally induced increase of $L$ at about $167 \mathrm{meV}$ for sample A, as shown in the inset of Fig. 1. Similar procedure was performed on the remaining three samples, with the activation energies ranging from 80 to $165 \mathrm{meV}$. No clear correlation between the activation energy and doping level was observed.

It is also shown in Fig. 1 that a nearly twofold increase in diffusion length is obtained after about 2 min of continuous electron irradiation at room temperature. As the sample temperature is raised, the rate of irradiation-induced diffusion length increase $R$ diminishes. The slower rates at elevated temperatures are attributed to the higher probability for the escape of the trapped electrons due to larger thermal energy. This process reestablishes the original recombination pathway and thus competes with irradiation-induced lifetime increase (see mechanism description above). The relationship between the rate $R$ and sample temperature can be modeled with the following expression: ${ }^{10}$

$$
R=R_{0} \exp \left(\frac{\Delta E_{A, I}}{k T}\right) \exp \left(-\frac{\Delta E_{A, T}}{2 k T}\right) .
$$

In Eq. (2), $R_{0}$ is a scaling constant, $\Delta E_{A, I}$ is the activation energy for the electron irradiation induced effect, and $\Delta E_{A, T}$ is the activation energy for the thermally induced diffusion length increase determined using Eq. (1).

$\Delta E_{A, I}$ for all four samples was determined from Eq. (2), accounting for the individual values of thermal activation energy, as shown in Fig. 2. This figure demonstrates that the

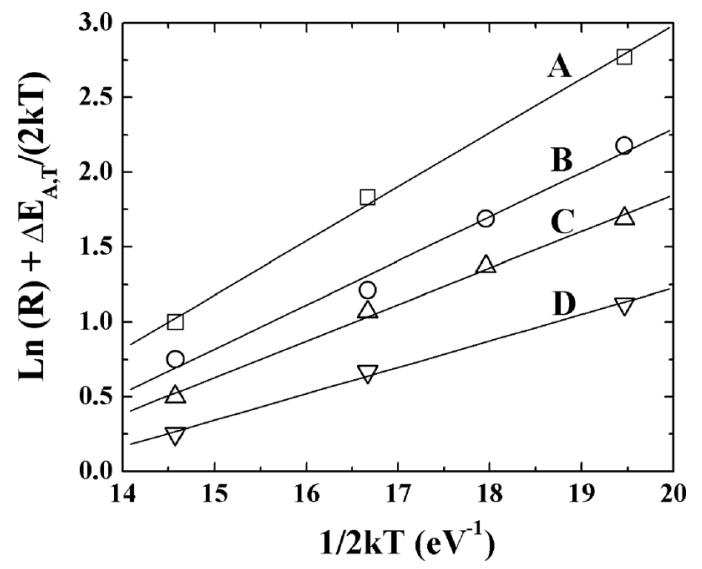

FIG. 2. Arrhenius plots of $R$ as a function of sample temperature for samples A-D and the fit based on Eq. (2) (solid lines). The plots were offset for clarity.

activation energy for irradiation-induced increase of $L$ shows a systematic dependence on the concentration of majority holes (see Table I) and therefore, on the doping level. The values for $\Delta E_{A, I}$ were found to be comparable to the generally accepted activation energy of $\mathrm{Mg}$ in $\mathrm{GaN}(\sim 200 \mathrm{meV})$. Since the former parameter has been previously correlated with the activation energy of dominant acceptors, both in GaN:Mg (Ref 11) and other systems, ${ }^{1,3,10,12}$ it is likely that this behavior is a manifestation of the concentration dependence of $\mathrm{Mg}$ acceptor activation energy. Furthermore, similar dependence was observed earlier for nonequilibrium carrier lifetime by temperature-dependent cathodoluminescence studies of $\mathrm{GaN}$ with different $\mathrm{Mg}$ concentrations. ${ }^{8}$

The decrease of acceptor activation energy with increased concentration is a common phenomenon in semiconductors $^{13-15}$ that has been attributed to a number of causes, including the formation of the band-tail states that extend into the forbidden gap, the broadening of the acceptor band in the gap, and the reduction of binding energy due to Coulomb interaction between the holes in the valence band and the ionized acceptor states. ${ }^{16}$ The relationship below is often used to describe this behavior,

$$
\Delta E_{A}\left(N_{A^{-}}\right)=\Delta E_{A}(0)-\alpha\left(N_{A^{-}}\right)^{1 / 3} .
$$

Here, $N_{A^{-}}$is the concentration of ionized acceptors, $\Delta E_{A}(0)$ is the ionization energy at very low doping levels, and $\alpha$ is a constant which accounts for geometrical factors as well as for the properties of the material and has the following form: ${ }^{15}$

$$
\alpha=\left(\Gamma(2 / 3) \frac{4 \pi}{3}\right)\left(\frac{q^{2}}{4 \pi \varepsilon}\right) .
$$

$\Gamma(2 / 3)(4 \pi / 3)$ is a geometric factor describing the probability of finding another ionized acceptor in the neighborhood of an ionized acceptor, ${ }^{14} q$ is elementary charge, and $\varepsilon$ is dielectric constant. The value of $\alpha$ according to Eq. (4) and taking $\varepsilon=9.5 \varepsilon_{0}$ for $\mathrm{GaN}$ (Ref. 15) was found to equal 3.3 $\times 10^{-8} \mathrm{eV} \mathrm{cm} . N_{A^{-}}$for each sample was calculated based on the assumption that $N_{A^{-}}-N_{D^{+}}=p$, where $N_{D^{+}}$is the density of ionized shallow donors and, based on the manufacturer's specification, is approximately $1 \times 10^{17} \mathrm{~cm}^{-3}$. Figure 3 shows that the values of $\Delta E_{A, I}$ follow the trend described by Eq. (3). Furthermore, the value of $\alpha$ obtained from the fit is $8.3 \times 10^{-8} \mathrm{eV} \mathrm{cm}$, which is comparable to that predicted by 


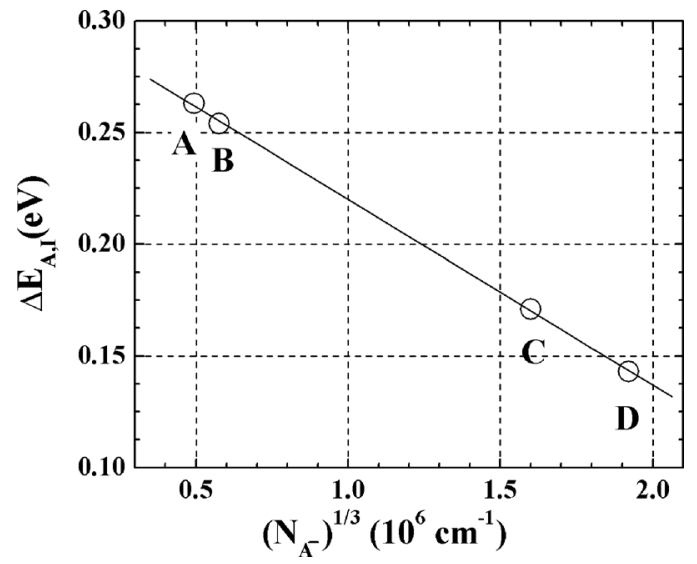

FIG. 3. Decrease of activation energy of electron irradiation effect as a function of ionized acceptor concentration and the linear fit (solid line) with Eq. (3).

Eq. (4) above. These findings further demonstrate that the irradiation-induced effects are linked to the electron trapping on nonionized $\mathrm{Mg}$ acceptor states.

In summary, variable-temperature minority carrier diffusion length measurements were used to study the role of doping levels in the effect of electron irradiation on minority carrier transport in a series of GaN:Mg samples. It was demonstrated that the activation energy of the irradiation-induced diffusion length increase diminishes with increasing carrier concentration. This behavior was found to be consistent with the concentration-dependent decrease in the $\mathrm{Mg}$ acceptor ionization energy, implying that trapping on $\mathrm{Mg}$ acceptor levels is a key step in the mechanism of the observed irradiation effect.
This work was supported in part by the National Science Foundation Grant No. (ECS 0422604 and DMR 04041638), the American Chemical Society Petroleum Research Fund Grant No. (40501-AC10), and NATO Science for Peace program (SfP 981939).

${ }^{1}$ W. Burdett, O. Lopatiuk, L. Chernyak, M. Hermann, M. Stutzmann, and M. Eickhoff, J. Appl. Phys. 96, 3556 (2004).

${ }^{2}$ L. Chernyak, A. Osinsky, V. Fuflyigin, and E. F. Schubert, Appl. Phys. Lett. 77, 875 (2000).

${ }^{3}$ O. Lopatiuk, W. Burdett, L. Chernyak, K. P. Ip, Y. W. Heo, D. P. Norton, S. J. Pearton, B. Hertog, P. P. Chow, and A. Osinsky, Appl. Phys. Lett. 86, 012105 (2005).

${ }^{4}$ O. Lopatiuk-Tirpak, L. Chernyak, F. X. Xiu, J. L. Liu, S. Jang, F. Ren, S. J. Pearton, A. Osinsky, and P. Chow, J. Appl. Phys. 100, 086101 (2006).

${ }^{5}$ L. Chernyak, G. Nootz, and A. Osinsky, Electron. Lett. 37, 922 (2001).

${ }^{6}$ O. Lopatiuk-Tirpak, L. Chernyak, L. J. Mandalapu, Z. Yang, J. L. Liu, K. Gartsman, Y. Feldman, and Z. Dashevsky, Appl. Phys. Lett. 89, 142114 (2006).

${ }^{7}$ L. Chernyak, A. Osinsky, and A. Schulte, Solid-State Electron. 45, 1687 (2001).

${ }^{8}$ O. Lopatiuk-Tirpak, L. Chernyak, Y. L. Wang, F. Ren, S. J. Pearton, K. Gartsman, and Y. Feldman, Appl. Phys. Lett. 90, 172111 (2007).

${ }^{9}$ L. Chernyak, A. Osinsky, H. Temkin, J. W. Yang, Q. Chen, and M. A. Khan, Appl. Phys. Lett. 69, 2531 (1996).

${ }^{10}$ O. Lopatiuk, L. Chernyak, A. Osinsky, and J. Q. Xie, Appl. Phys. Lett. 87, 214110 (2005).

${ }^{11}$ W. Burdett, A. Osinsky, V. Kotlyarov, P. Chow, A. Dabiran, and L. Chernyak, Solid-State Electron. 47, 931 (2003).

${ }^{12}$ O. Lopatiuk, A. Osinsky, A. Dabiran, K. Gartsman, I. Feldman, and L. Chernyak, Solid-State Electron. 49, 1662 (2005).

${ }^{13}$ P. P. Debye and E. M. Comwell, Phys. Rev. 93, 639 (1954).

${ }^{14}$ W. Gotz, R. S. Kern, C. H. Chen, H. Liu, D. A. Steigerwald, and R. M. Fletcher, Mater. Sci. Eng., B 59, 211 (1999).

${ }^{15}$ P. Kozodoy, H. L. Xing, S. P. DenBaars, U. K. Mishra, A. Saxler, R. Perrin, S. Elhamri, and W. C. Mitchel, J. Appl. Phys. 87, 1832 (2000).

${ }^{16}$ M. G. Cheong, K. S. Kim, C. S. Kim, R. J. Choi, H. S. Yoon, N. W. Namgung, E. K. Suh, and H. J. Lee, Appl. Phys. Lett. 80, 1001 (2002). 